\title{
Pre-edges in oxygen (1s) x-ray absorption spectra: A spectral indicator for electron hole depletion and transport blocking in iron perovskites
}

\author{
Artur Braun, ${ }^{1, a)}$ Defne Bayraktar, ${ }^{1}$ Selma Erat, ${ }^{1,2}$ Ashley S. Harvey, ${ }^{2}$ Daniel Beckel, ${ }^{2}$ \\ John A. Purton, ${ }^{3}$ Peter Holtappels, ${ }^{1}$ Ludwig J. Gauckler, ${ }^{2}$ and Thomas Graule ${ }^{1}$ \\ ${ }^{1}$ Laboratory for High Performance Ceramics, EMPA-Swiss Federal Laboratories for Materials Testing \& \\ Research, CH-8600 Dübendorf, Switzerland \\ ${ }^{2}$ Department of Nonmetallic Inorganic Materials, ETH Zürich-Swiss Federal Institute of Technology, CH- \\ 8053 Zürich, Switzerland \\ ${ }^{3}$ Synchrotron Radiation Source, Daresbury Laboratory, Warrington WA4 4AD, United Kingdom
}

(Received 27 December 2008; accepted 30 March 2009; published online 19 May 2009)

The $e_{g} \uparrow /\left(t_{2 g} \downarrow+e_{g} \downarrow\right)$ band ratio in cation-substituted La-Fe oxides is identified in O (1s) x-ray absorption spectra as a linear spectral indicator for conducting electron holes. The $t_{2 g} \downarrow$ and $e_{g} \downarrow$ bands act as a conductivity inhibitor by ferromagnetic double exchange coupling on the $e_{g} \uparrow$ electron. Disorder induced by substitution appears to modulate the hole conduction such that an exponential relation is found between the conductivity and the $e_{g} \uparrow /\left(t_{2 g} \downarrow+e_{g} \downarrow\right)$ ratio and hole concentration. The quantitative correlation of conductivity and x-ray absorption spectra from heterovalent-substituted $\mathrm{LaFeO}_{3}$ lets substitution-driven metal insulator transitions appear in a new light. () 2009 American Institute of Physics. [DOI: 10.1063/1.3122926]

Rare-earth transition metal oxides display a manifold of technologically relevant functionalities, which are controlled by the oxygen ion and hybridization of $\mathrm{O}(2 p)$ states with the transition metal $d$ states. Control of transport mechanisms, which are vital for energy materials, can be obtained with cation doping and tuning oxygen stoichiometry. For instance, $\mathrm{Ta}$ and $\mathrm{Ti}$ in $\mathrm{La}-\mathrm{Sr}-\mathrm{Fe}$ (LSF) oxides are interesting for catalytic oxygen membranes, ${ }^{1} \mathrm{Ta}$ is a hole depletion agent in photocatalysts, ${ }^{2}$ and $\mathrm{Ti}$ suppresses leakage currents in high temperature tantalum capacitors. ${ }^{3}$

The role of electron holes as charge carriers and their detection in the pre-edge of oxygen x-ray absorption spectra (XAS) has been recognized. But the pre-edges withstood quantitative treatment so far.

Heterovalent substitution of $\mathrm{La}^{3+}$ in the charge transfer insulator $\mathrm{LaFeO}_{3}$ by $\mathrm{Sr}^{2+}$ creates electron hole states with $\mathrm{O}(2 p)$ character near the Fermi energy $E_{F} \cdot{ }^{4-7}$ In addition to hole doping, the increasing radius from $\mathrm{La}$ to $\mathrm{Sr}$ decreases the rhombohedral lattice distortion, which in turn increases the hybridization of the $\mathrm{O}(2 p)-\mathrm{Fe}(3 d)$ states and, thus, the one-electron band width. ${ }^{8}$

The conductivity of $\mathrm{La}_{1-x} \mathrm{Sr}_{x} \mathrm{FeO}_{3}$ increases with increasing $x, \mathrm{SrFeO}_{3}$ displaying metallic conductivity. ${ }^{6,8}$ Charge balance is maintained by adjustment of the $\mathrm{Fe}$ oxidation state and oxygen deficiency $\delta$. While the formal valence is $\mathrm{Fe}^{3+}$ in $\mathrm{LaFeO}_{3}\left(\mathrm{Fe}^{4+}\right.$ in $\left.\mathrm{SrFeO}_{3}\right)$, the actual relative $\mathrm{Fe}^{4+}$ concentration in $\mathrm{SrFeO}_{3-\delta}$ may be below $40 \%$. With every $\mathrm{Fe}^{4+}$ replacing one $\mathrm{Fe}^{3+}$, one electron charge carrier hole is created. ${ }^{9}$ Our starting reference $\mathrm{La}_{0.5} \mathrm{Sr}_{0.5} \mathrm{FeO}_{3-\delta}$ (LSF50) with maximum conductivity ${ }^{7,10}$ is then doped with $10 \% \mathrm{Ta}$, $20 \% \mathrm{Ta}$, and $20 \% \mathrm{Ti}$. Competition between various mixed valence ions on $A\left(B B^{*}\right) \mathrm{O}_{3}$ type perovskites is attracting increasingly interest. ${ }^{11}$

The four-point dc conductivity (Burster Resistomat, accuracy $0.03 \%$ of reading; resolution $10 \mu \Omega$ ) of phase pureLSF50 and substituted LSF with $R-3 c$ symmetry (Table I) was measured on sintered bars with density $>95 \%$ of the theoretical density. ${ }^{1}$ The polaron activation energy $E_{a}$ was determined by a least square fit of $\sigma(T)$ $=\sigma_{0} / T \exp \left(E_{a} / k T\right)$ from 300 to $700 \mathrm{~K}$. For details on structure and properties of LSF see Refs. 7 and 12. LSF50 has an average formal valence of 3.5 on iron and a formal hole concentration of $\tau=\mathrm{Fe}^{4+} / \mathrm{Fe}^{3+}=1$. The gravimetrically obtained $\delta$ served to calculate the actual hole concentration $\tau$ (Table I).

Conductivity $\sigma$ at $307 \mathrm{~K}$ and activation energy $E_{a}$ are listed in Table I. By $20 \%$ substitution of $\mathrm{Fe}$ with $\mathrm{Ta}$ $\left(\mathrm{La}_{0.5} \mathrm{Sr}_{0.5} \mathrm{Fe}_{0.8} \mathrm{Ta}_{0.2} \mathrm{O}_{3-\delta} \mathrm{LSFTa} 20\right), \sigma$ decreases by three orders of magnitude, while $E_{a}$ increases by a factor of 2.3 . LSF50 has the overall highest conductivity at about $300 \mathrm{~K}$, systematically followed by $\mathrm{La}_{0.5} \mathrm{Sr}_{0.5} \mathrm{Fe}_{0.8} \mathrm{Ti}_{0.2} \mathrm{O}_{3-\delta}$, $\mathrm{La}_{0.5} \mathrm{Sr}_{0.5} \mathrm{Fe}_{0.9} \mathrm{Ta}_{0.1} \mathrm{O}_{3-\delta}$, and $\mathrm{La}_{0.5} \mathrm{Sr}_{0.5} \mathrm{Fe}_{0.8} \mathrm{Ta}_{0.2} \mathrm{O}_{3-\delta}$, with

TABLE I. Oxygen deficiency $\delta$, experimental hole concentration $\tau$, conductivity $\sigma$, polaron activation energy $E_{a}$, branching ratio $Q$, and spin-orbit splitting $\Delta$.

\begin{tabular}{lcccccc}
\hline \hline Stoichiometry & $\delta$ & $\tau$ & $\begin{array}{c}\sigma \\
(\mathrm{S} / \mathrm{cm})\end{array}$ & $\begin{array}{c}E_{a} \\
(\mathrm{meV})\end{array}$ & $Q\left[L_{3} /\left(L_{2}+L_{3}\right)\right]$ & $\begin{array}{c}\text { Spin-orbit splitting } \Delta \\
(\mathrm{eV})\end{array}$ \\
\hline $\mathrm{La}_{0.5} \mathrm{Sr}_{0.5} \mathrm{FeO}_{3-\delta}$ & 0.078 & 0.52 & 50 & 180 & 0.67 & 12.85 \\
$\mathrm{La}_{0.5} \mathrm{Sr}_{0.5} \mathrm{Fe}_{0.8} \mathrm{Ti}_{0.2} \mathrm{O}_{3-\delta}$ & 0.063 & 0.28 & 2.28 & 262 & 0.68 & 12.97 \\
$\mathrm{La}_{0.5} \mathrm{Sr}_{0.5} \mathrm{Fe}_{0.9} \mathrm{Ta}_{0.1} \mathrm{O}_{3-\delta}$ & 0.058 & 0.26 & 0.82 & 342 & 0.66 & 12.86 \\
\hline
\end{tabular}

\footnotetext{
${ }^{a)}$ Electronic mail: artur.braun@alumni.ethz.ch.
} 


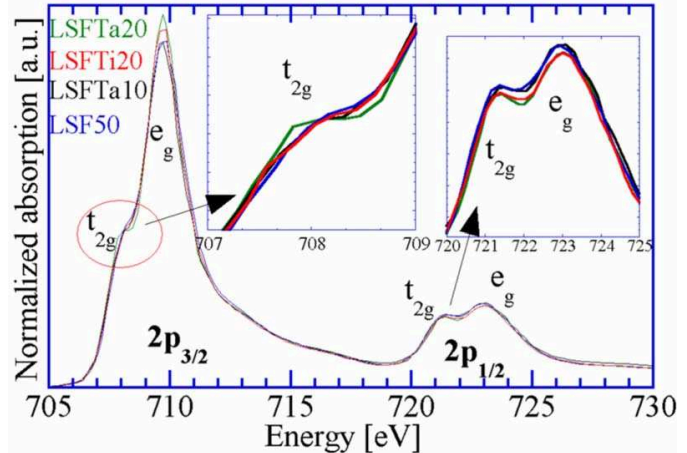

FIG. 1. (Color online) Normalized Fe $L$-edge spectra.

the lowest $\sigma$. Comparison of $\sigma$ and $\tau$ (Table I) reveals direct correlation between $\sigma$ and substitution level.

Soft XAS were recorded at $300 \mathrm{~K}$ and $5 \times 10^{-10}$ Torr at Daresbury Laboratory Synchrotron Radiation Source (beamline 1.1), United Kingdom. Figure 1 shows the area normalized $\mathrm{Fe}(2 p)$ absorption spectra corrected for the chemical shift. Differences of the overlaid spectra can be made out as a minute variation of the $2 p_{3 / 2} t_{2 g}$ shoulder and the height of the $e_{g}$ peak, and the different height of the $2 p_{1 / 2}$ peak. The multiplet structure of $3 d$ metals such as iron and its x-ray spectroscopic assessment are generally well understood ${ }^{5,13}$ and provide a unique probe for the local spin moment of magnetic Fe ${ }^{14}$ The degeneracy of the Fe $(2 p)$ core hole level is lifted by spin-orbit coupling, resulting in the $2 p_{3 / 2}$ and $2 p_{1 / 2}$ multiplets at around 708 and $722 \mathrm{eV}$. Their centroids are separated by around $13 \mathrm{eV}$ (Table I). The octahedral crystal field lifts the degeneracy of the $2 p_{3 / 2}$ and $2 p_{1 / 2}$ levels so that two levels with $t_{2 g}$ and $e_{g}$ symmetry are created, as indicated by the two structures at about 707 and $708.5 \mathrm{eV}$ and at 720.5 and $722 \mathrm{eV}$.

The chemical shift of the white lines revealed that the average oxidation state of the $\mathrm{Fe}$ varies from sample to sample. LSFTa20 contains - due to charge compensation by $\mathrm{Ta}^{5+}$ - only $\mathrm{Fe}^{3+}$ and thus, has the lowest $\mathrm{Fe}$ oxidation state and no holes. With only high spin $\mathrm{Fe}^{3+}$ in $3 d^{5}$ configurations LSFTa20 has the largest multiplet branching ratio $Q$ $=L_{3} /\left(L_{2}+L_{3}\right)=0.70$ (Table I). ${ }^{15}$ Due to the larger cation size of $\mathrm{Ti}^{4+}$ and $\mathrm{Ta}^{5+}$ compared to $\mathrm{Fe}^{4+}$, the unit cell of LSF expands upon substitution with $\mathrm{Ti}$ and $\mathrm{Ta},{ }^{1}$ possibly providing the remaining $\mathrm{Fe}$ ions more space for parallel spins. In line with Ref. 5, we find that $\mathrm{Fe}$ remains essentially in the ferromagnetic high spin $3 d^{5}$ configuration $\left(t_{2 g}^{3} e_{g}^{2}\right)$.

The prepeak of oxygen $K$-edge spectra due to doped holes in $A$-site substituted LaSrMn-oxide correlates well with the conductivity. ${ }^{16}$ We extend that study to $B$-site substituted LSF. For deeper insight in the transport mechanism, we recorded O (1s) XAS (Fig. 2), which represent to a good approximation the unoccupied oxygen $p$ projected density of states. ${ }^{17}$ Since the other orbitals are hybridized with the $\mathrm{O}(2 p)$ orbitals, the spectra also reflect empty $\mathrm{Fe}(3 d)$ and $\mathrm{La}(5 d)$ bands. $\mathrm{LaFeO}_{3}$ forms spin-down $t_{2 g} \downarrow$ and $e_{g} \downarrow$ peaks at around 528 and $531 \mathrm{eV}$. They represent mixed $\mathrm{O}(2 p)-\mathrm{Fe}(3 d)$ states subject to the ligand field splitting of $\Delta \approx 1.1 \mathrm{eV}$ in the $\mathrm{FeO}_{6}$ octahedra, and hence form $t_{2 g} \downarrow$ and $e_{g} \downarrow$ states in the band-gap. ${ }^{5,6,18}$ The extra peak from hole doping has spin-up $e_{g} \uparrow$ nature. ${ }^{19}$ The pre-edge features between 526 and $531 \mathrm{eV}$ are deconvoluted into the well-known $t_{2 g} \downarrow$ and $e_{g} \downarrow$ bands, and the extra $e_{g} \uparrow$ band for the doped hole states at around $528 \mathrm{eV}$ (Fig. 2). The sharp peak at $531.6 \mathrm{eV}$ from the carbon tape, on which the samples were dispersed, confirms energy alignment of the spectra. Subtraction of this peak, as exercised for LSF50, brings the pre-edge features back in the well-known shape. ${ }^{19}$ With systematic differences depending on the doping situation, we notice considerable variation of the prepeak intensities. The observation that for LSF50 the intensity of one $e_{g} \uparrow$ hole counts as much as that of the five spin-down holes might be explained by that the doped hole is localized on oxygen (hence close to $100 \%$ contributing) and the ten spin-down holes are mainly iron state with some admixture of oxygen character. According to Ref. 5, holes from $A$-site substitution go to states of primarily oxygen character, and the main component of the ground state in LSF50 is $3 d^{5} \underline{L}$ (ligand hole $\underline{L}$ ) and not $3 d^{4}$. In their study, and in the study by Wadati $e t a l .,{ }^{19}$ the $e_{g} \uparrow$ peak grows with increasing $\mathrm{Sr}$ content and permits quantitative consideration of hole doping by substitution at the $A$ site, which to some extent was already exercised in LSM. ${ }^{16}$ Homovalent $B$-site substitution may cause a mismatch in the lattice, redistributing empty hole states near $E_{F}$, thus improving the conductivity. ${ }^{4}$ Here, where the $B$-site is substituted with the supervalent $\mathrm{Ta}$ and $\mathrm{Ti}$, we observe the opposite effect: blocking the conductivity. Change in the $e_{g} \uparrow$ intensity alone does not permit systematic conclusions about the material, much less the conductivity. ${ }^{5}$ As will be shown, the ratio $S$ $=e_{g} \uparrow /\left(t_{2 g} \downarrow+e_{g} \downarrow\right)$ correlates better with $\tau$ and $\sigma$.

Correlation of $\sigma$ and $S$ is shown in Fig. 3. Filled circles denote our $B$-site doping series for Ta, including the nondoped LSF. The conductivity increases exponentially with increasing $S$, as shown by the fit.

It is interesting to discuss $S$ in terms of $\tau$. The data points (filled circles and squares) show a linear correlation (linear fit) of the dimensionless form $S(\tau)=S_{0}+a \cdot \tau$. Due to the mutual relationship between $S, \sigma$, and $\tau$ we can (not shown here) plot $\sigma$ versus $\tau$ and fit it with an exponential of the form $\sigma \sim \sigma_{0} \exp (U \tau)$. Our samples from $B$-site substitution have nonzero ratio $S_{0}$ for vanishing $\tau$. Certainly, LSFTi20 has some residual conductivity even in the absence of holes, and the corresponding spectral intensity near $E_{F}$ and the corresponding conductivity derive not only from hole states. Also, we cannot entirely rule out that this intensity is caused
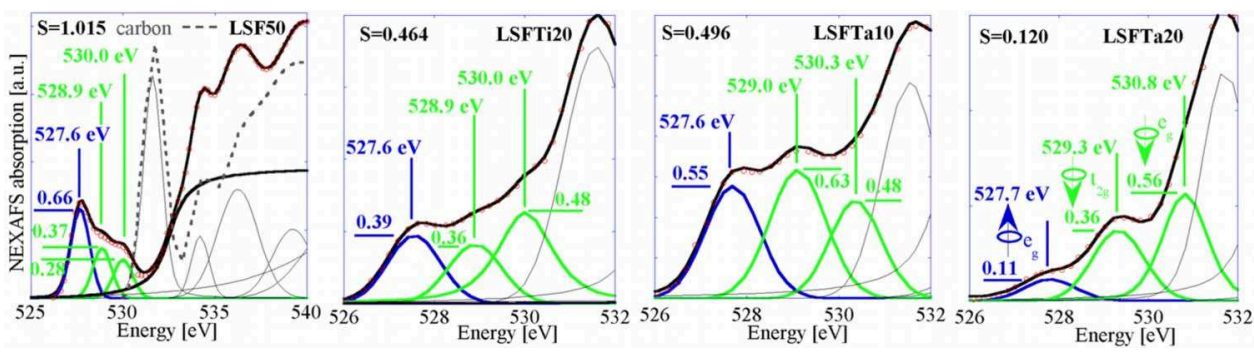

FIG. 2. (Color online) Deconvoluted $\mathrm{O}(1 s)$ spectra with prepeak near $E_{F}$. Numerals indicate peak position and spectral weight. 


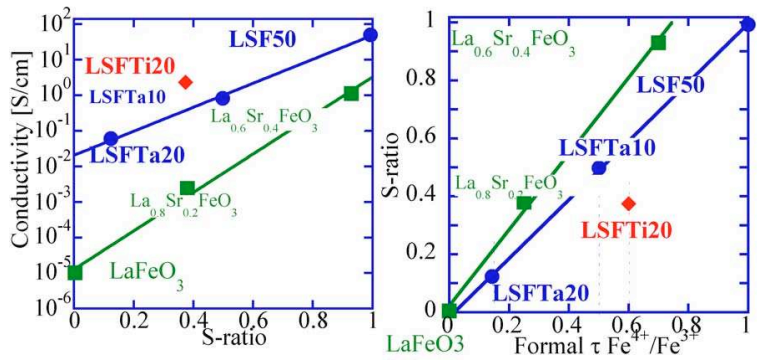

FIG. 3. (Color online) Correlation and exponential least square fit of $\sigma$ and $S$-ratio. Circles are for $\mathrm{La}_{0.5} \mathrm{Sr}_{0.5} \mathrm{FeO}_{3-\delta}, \mathrm{La}_{0.5} \mathrm{Sr}_{0.5} \mathrm{Fe}_{0.9} \mathrm{Ta}_{0.1} \mathrm{O}_{3-\delta}$, and $\mathrm{La}_{0.5} \mathrm{Sr}_{0.5} \mathrm{Fe}_{0.8} \mathrm{Ta}_{0.2} \mathrm{O}_{3-\delta}$. Squares are data from Ref. 19.

by $\mathrm{O}(2 p)-\mathrm{Ta}(5 d)$ mixed states, as may be the case for other substituted samples. On the other hand, addition of Ti and Ta will slightly expand the unit cell, a situation which will generally not favor an increase in $\sigma$ and, as observed with $20 \% \mathrm{Ta}$, may even shift the pre-edge band to slightly higher energies.

Since the nonsubstituted LSF50 sample fits too in our proposed scheme, we believe that $S$ as an indicator for conductivity is more general than for $B$-site substitution only. To support this hypothesis, we plotted these $S$-ratios for published data ${ }^{19}$ (an $A$-site substitution study), i.e $\mathrm{La}_{0.6} \mathrm{Sr}_{0.4} \mathrm{FeO}_{3}$, $\mathrm{La}_{0.8} \mathrm{Sr}_{0.2} \mathrm{FeO}_{3}$, and $\mathrm{LaFeO}_{3}$ in Fig. 3 as well. These ratios can be expressed as a linear function in $\tau$. Note that we now have a set of $A$-site and $B$-site substituted LSF materials with a linear relation of the $S$ - ratio and relative $\tau$ (Fig 3). In both cases the unit cell volume increases. ${ }^{9}$ By substitution of the $B$ cation rather than the $A$ cation, we become more sensitive to subtleties of the $\mathrm{Fe}-\mathrm{O}-(\mathrm{Fe}, \mathrm{Ta}, \mathrm{Ti}, \ldots)$ superexchange unit, which ultimately provides-or blocks-the transport, particularly since $A$ cations usually do not transport).

Why should $t_{2 g} \downarrow$ and $e_{g} \downarrow$, which are more remote from $E_{F}$ than the $e_{g} \uparrow$ band, have any relevance to the transport properties? The $t_{2 g} \downarrow$ orbitals are localized and do not point toward the neighboring ligands, whereas the more delocalized $e_{g} \uparrow$ bands do so and hence basically operate as switches for the charge transfer across the superexchange unit. Resolution is provided by Zener's ${ }^{20}$ double exchange, which ferromagnetically couples the $t_{2 g} \downarrow$ spins with the mobile $e_{g} \uparrow$ electrons. In this context, it is interesting to note that our samples with the highest $\sigma$ and smallest $E_{a}$ also have the smallest branching ratios $Q$ (Table I, see also Fig. 6 in Ref. 7). The more remote $e_{g} \downarrow$ peak in the $t_{2 g} \downarrow$ and $e_{g} \downarrow$ pair seems to be of little influence for $\sigma$ within the accuracy of our data.

$\mathrm{Fe}^{4+}$ with $3 d^{5} L$ configuration ${ }^{5}$ has octahedral $d^{2} s p^{3}$ coordination, whereas $\mathrm{Fe}^{3+}$ with a $3 d^{5}$ configuration prefers tetrahedral coordination. Depletion of $\mathrm{Fe}^{4+}$ with increasing $\mathrm{Ta}$ or $\mathrm{Ti}$ substitution, and resulting enrichment of $\mathrm{Fe}^{3+}$, causes coordination from octahedral to tetrahedral, which consequently manifests in a depletion of the conducting $e_{g} \uparrow$ bands, which require octahedral coordination. ${ }^{7}$

The observed exponential relation between $\tau$ and $\sigma$, and the linear relation between $\tau$ and $S$, can be written in simple mathematical terms, $\sigma \sim \exp \left\{U\left[e_{g} \uparrow /\left(t_{2 g} \downarrow+e_{g} \downarrow\right)\right]\right\}$, with specific constant $U$.

The fact that the decrease of $\sigma$ is exponential, while the hole variation and the spectral changes are linear, suggests that the hole concentration per se cannot account for the entire conductivity loss. Such is found for Ta doping on $\mathrm{Na}_{x} \mathrm{WO}_{3}{ }^{21}$ and for $\mathrm{Ni} / \mathrm{Fe}$ substitution on $\mathrm{LaNi}_{1-x} \mathrm{Fe}_{x} \mathrm{O}_{3}$. ${ }^{22}$
We speculate that substitution disorder and consequential percolation may be an additional cause for the conductivity decrease. Okunev et $\mathrm{al}^{23}$ find an exponential relationship between $\sigma$ and the distance of nonconducting clusters on LaSrMn- oxide, for example. We envision a similar situation here, with a ratio of more conducting $e_{g} \uparrow$ and less conducting $t_{2 g} \downarrow$ species, which is the argument of and modulated by an exponential. Since $e_{g} \downarrow$ is remote from $E_{F}$, its influence on the conductivity is negligible.

This study provides quantitative results that clarify and unify our understanding of substitution mechanisms. The combination of transport measurements and x-ray spectroscopy, particularly that of $\mathrm{O}(1 s)$ for unoccupied states, holds promise to tell whether conductivity changes are related to hole doping or depletion, or to changes in the band structure due to lattice structure alterations.

Financial support by the Swiss National Science Foundation Project No. 200021-100674/1 and the European Commission under Grant No. MIRG-CT-2006-042095. The Science and Technology Facilities Council of the U.K. are acknowledged for beamtime at the Synchrotron Radiation Source, Project No. 47093.

${ }^{1}$ D. Bayraktar, S. Diethelm, P. Holtappels, T. Graule, and J. Van herle, J. Solid State Electrochem. 10, 589 (2006).

${ }^{2}$ T. Ishii, H. Kato, and A. Kudo, J. Photochem. Photobiol., A 163, 181 (2004)

${ }^{3}$ W. S. Lau, T. S. Tan, and P. Babu, Appl. Phys. Lett. 90, 112903 (2007). ${ }^{4}$ D. D. Sarma, O. Rader, T. Kachel, A. Chainani, M. Mathew, K. Holldack, W. Gudat, and W. Eberhardt, Phys. Rev. B 49, 14238 (1994).

${ }^{5}$ M. Abbate, F. M. F. de Groot, J. C. Fuggle, A. Fujimori, O. Strebel, F. Lopez, M. Domke, G. Kaindl, G. A. Sawatzky, M. Takano, Y. Takeda, H. Eisaki, and S. Uchida, Phys. Rev. B 46, 4511 (1992).

${ }^{6}$ H. Wadati, D. Kobayashi, H. Kumigashira, K. Okazaki, T. Mizokawa, A. Fujimori, K. Horiba, M. Oshima, N. Hamada, M. Lippmaa, M. Kawasaki, and H. Koinuma, Phys. Rev. B 71, 035108 (2005).

${ }^{7}$ J.-C. Grenier, N. Ea, M. Pouchard, and M. M. Abou-Sekkina, Mater. Res. Bull. 19, 1301 (1984).

${ }^{8}$ S. K. Park, T. Ishikawa, Y. Tokura, J. Q. Li, and Y. Matsui, Phys. Rev. B 60, 10788 (1999).

${ }^{9}$ X.-D. Zhou, Q. Cai, J. Yang, M. Kim, W. B. Yelon, W. J. James, J. Appl. Phys. 97, 10C314 (2005)

${ }^{10}$ M. V. Patrakeev, J. A. Bahteeva, E. B. Mitberg, I. A. Leonidov, V. L. Kozhevnikov, and K. R. Poeppelmeier, J. Solid State Chem. 172, 219 (2003).

${ }^{11}$ J.-S. Kang, H. J. Lee, G. Kim, D. H. Kim, B. Dabrowski, S. Kolesnik, Hangil Lee, J. -Y. Kim, and B. I. Min, Phys. Rev. B 78, 054434 (2008).

${ }^{12}$ S. E. Dann, D. B. Currie, M. T. Weller, M. F. Thomas, and A. D. AlRawwas, J. Solid State Chem. 109, 134 (1994).

${ }^{13}$ F. de Groot and A. Kotani, Core Level Spectroscopy of Solids (CRC, Boca Raton, 2007).

${ }^{14}$ C. S. Fadley, D. A. Shirley, A. J. Freeman, and P. S. Bagus, Phys. Rev. Lett. 23, 1397 (1969).

${ }^{15}$ B. T. Thole and G. van der Laan, Phys. Rev. B 38, 3158 (1988).

${ }^{16}$ H. L. Ju, H. -C. Sohn, and K. M. Krishnan, Phys. Rev. Lett. 79, 3230 (1997).

${ }^{17}$ F. M. F. de Groot, M. Grioni, J. C. Fuggle, J. Ghijsen, G. A. Sawatzky, and H. Petersen, Phys. Rev. B 40, 5715 (1989).

${ }^{18}$ Z. Y. Wu, S. Gota, F. Jollet, M. Pollak, M. Gautier-Soyer, and C. R. Natoli, Phys. Rev. B 55, 2570 (1997).

${ }^{19}$ H. Wadati, A. Chikamatsu, R. Hashimoto, M. Takizawa, H. Kumigashira, A. Fujimori, M. Oshima, M. Lippmaa, M. Kawasaki, and H. Koinuma, J. Phys. Soc. Jpn. 75, 054704 (2006).

${ }^{20}$ C. Zener, Phys. Rev. 82, 403 (1951).

${ }^{21}$ A. K. Raychaudhuri, Phys. Rev. B 44, 8572 (1991).

${ }^{22}$ D. D. Sarma, D. D. Sarma, A. Chainani, S. R. Krishnakumar, E. Vescovo, C. Carbone, W. Eberhardt, O. Rader, Ch. Jung, Ch. Hellwig, W. Gudat, H. Srikanth, and A. K. Raychaudhuri, Phys. Rev. Lett. 80, 4004 (1998).

${ }^{23}$ V. D. Okunev, R. Szymczak, M. Baran, H. Szymczak, and P. Gierlowski, Phys. Rev. B 74, 014404 (2006). 